\title{
HMGA1 and HMGA2 expression and comparative analyses of HMGA2, Lin28 and let-7 miRNAs in oral squamous cell carcinoma
}

\author{
Katharina Anna Sterenczak', Andre Eckardt², Andreas Kampmann², Saskia Willenbrock', Nina Eberle, \\ Florian Länger ${ }^{3}$, Sven Kleinschmidt ${ }^{4}$, Marion Hewicker-Trautwein ${ }^{4}$, Hans Kreipe ${ }^{3}$, Ingo Nolte ${ }^{1 *}$, \\ Hugo Murua Escobar ${ }^{1,5}$ and Nils Claudius Gellrich ${ }^{2}$
}

\begin{abstract}
Background: Humans and dogs are affected by squamous cell carcinomas of the oral cavity (OSCC) in a considerably high frequency. The high mobility group A2 (HMGA2) protein was found to be highly expressed in human OSCC and its expression was suggested to act as a useful predictive and prognostic tool in clinical management of oral carcinomas. Herein the expression of HMGA2 and its sister gene HMGA1 were analysed within human and canine OSCC samples. Additionally, the HMGA negatively regulating miRNAs of the let-7 family as well as the let-7 regulating gene Lin28 were also comparatively analysed. Deregulations of either one of these members could affect the progression of human and canine OSCC.
\end{abstract}

Methods: Expression levels of HMGA1, HMGA2, Lin28, let-7a and mir-98 were analysed via relative QPCR in primary human and canine OSCC, thereof derived cell lines and non-neoplastic samples. Additionally, comparative HMGA2 protein expression was analysed by immunohistochemistry.

Results: In both species, a significant up-regulation of the HMGA2 gene was found within the neoplastic samples while HMGA1 expression did not show significant deregulations. Comparative analyses showed down-regulation of mir-98 in human samples and up-regulation of let-7a and mir-98 in canine neoplastic samples. HMGA2 immunostainings showed higher intensities within the invasive front of the tumours than in the centre of the tumour in both species.

Conclusions: HMGA2 could potentially serve as tumour marker in both species while HMGA1 might play a minor role in OSCC progression. Comparative studies indicate an inverse correlation of HMGA2 and mir-98 expression in human samples whereas in dogs no such characteristic could be found.

Keywords: Squamous cell carcinoma, HMGA1, HMGA2, let-7, mir-98, Lin28, Animal model, Dogs, Comparative oncology

\section{Background}

Oral cancer is the eighth most frequent cancer worldwide with even higher frequencies in developing than in developed countries [1]. Furthermore, men develop twice as frequent oral cancer than women and more than $95 \%$ of the carcinomas are of the squamous cell type. The standard treatment consists of surgery and/or radiation with additional chemotherapy in advanced stages of the disease. Tobacco and alcohol are regarded as the

\footnotetext{
* Correspondence: Ingo.Nolte@tiho-hannover.de

'Small Animal Clinic, University of Veterinary Medicine Hannover, Bünteweg 9, 30559 Hannover, Germany

Full list of author information is available at the end of the article
}

major risk factors for oral cancers but also infection with Human Papilloma Virus (HPV) are associated with a subset of head and neck cancers [2].

In dogs, oral cancer is the fourth most common cancer overall [3]. Similarly to humans, male dogs have a 2.4 times higher risk of developing oropharyngeal malignancies compared to female dogs and the tumours are staged similarly to those in humans but only $17-25 \%$ of the carcinomas are of the squamous cell type [3]. The risk for metastatic disease is site dependent with a higher metastatic potential in caudal tongue and tonsils and a lower metastatic rate in the rostral oral cavity [3]. Surgery and radiation are the most common treatment 
modalities and the mean survival time (MST) after surgery or radiation therapy is reported to lie between 26-36 months [3]. In humans, surgery and radiation are also the most common treatment modalities resulting in estimated overall 5-years survival rates for cancers of the oral cavity/ pharynx and larynx between 58.3\% and 64.5\% [4-6].

Due to the heterogeneity of head and neck tumours with different site specific prognosis and survival, the integration of multiple selected prognostic tumour markers in association with the histopathologic features is advocated for risk assessment. The search for biomarkers includes evaluation of tumour tissues and surrogate material by molecular, genomic and phenotypic means [7].

The high mobility group (HMG) protein A family consists of two members HMGA1 and HMGA2 encoding thee major proteins: HMGA1a, HMGA1b, and HMGA2. The expression of HMGA1 and HMGA2 is high during embryogenesis and strongly reduced to very low, hardly detectable levels in adult tissues [8]. Re-expression in adult tissues was described in several human and canine neoplasias as cancers of the prostate and colorectum as well as lymphomas and non-small cell lung cancer [8-10].

Concerning human oral carcinoma, analysis of HMGA2 expression was reported to be found significantly over-expressed in carcinoma tissues when compared to non neoplastic tissues [11]. Immunohistochemical localisation showed that HMGA2 protein was localised at the invasive front of oral carcinomas leading to the conclusion that HMGA2 immunostaining could be a prognostic determinant in stratifying patients into risk groups [11]. Analysis of HMGA1a and HMGA1b expression showed different findings reporting no significant expression deregulations [11] and increased expression in head and neck carcinomas, when compared to healthy mucosa samples [12].

HMGA2 expression was shown to be partly regulated by the let-7 miRNA family member mir-98 in head and neck squamous cell carcinoma cell lines [13]. Studies analysing $H M G A$ and let-7 expression in retinoblastomas and gastroenteropancreatic neuroendocrine tumors revealed a HMGA over expression accompanied by a down-regulation of let-7 [14,15].

Let-7 micro RNAs themselves are regulated posttranscriptionally by the LIN28 and LIN28B proteins encoded by the $\operatorname{Lin} 28$ gene [16-19]. Accordingly, overexpression of $\operatorname{Lin} 28$ was found to be linked to a repression of let-7 family miRNAs and a combined down-regulation of let-7 and up-regulation of $\operatorname{Lin} 28$ was reported in human neoplasias [20]. Interestingly a recent study analysing OSCCs reported increased expression of Lin $28 a$ and Lin $28 b$. Thereby, the increased levels of Lin $28 \mathrm{~b}$ could be associated with poor prognosis [21].

In summary, the Lin 28 - let-7 -HMGA regulatory pathway and deregulations of either one of these members or of all involved proteins and miRNAs could have an effect on the progression and pathogeneses of human and canine OSCC. Thus, in our study we investigated the expression levels of HMGA1, HMGA2, $\operatorname{Lin} 28$, let-7 a and mir-98 via relative real time PCR in human and canine non-neoplastic and tumour tissue samples and human and canine cell lines which derived from primary OSCCs.

\section{Methods}

\section{Tissue samples obtained from human patients}

This study included human squamous cell carcinoma, non neoplastic controls, and tumour derived cell line samples which were obtained from 12 patients ( 9 male, 3 female, age 20-71 years) who underwent surgery at the Department of Oral and Maxillofacial Surgery, Hannover Medical School. Ethical approval and informed patient consent was obtained for all patients. This study was approved by the local ethics committee at the Hannover Medical School (Ref No. 984-2011). No patients had received preoperative chemotherapy or radiotherapy. The tumours (patients 2-12) were staged according to TNM staging system and were classified as follows: patient 2- pT4apN1, patient 3- pT1pN0, patient 4- rpT0 rpN2b R2 M1, patient 5- pT3pN0, patient 6- pT4apN0, patient 7- pT4apN1, patient 8- pT4apN2b, patient 9- pT4apN2c, patient 10- pT4apN2b, patient 11- pT2pN0, and patient 12-pT4 pNo.

\section{Tissue samples obtained from canine patients}

Seven canine tumour and two healthy control samples (five female, four male) were used covering seven breeds: Boxer, Fox Terrier, Irish Terrier, Landseer, Retriever, Sheltie ( $\mathrm{n}=1$ respectively), and three Mixed-breeds. Age ranged between a half year and eleven years. Samples derived from the maxilla (4), tongue (2), mandible (1), palate (1), and pharynx (1). All tumours were analysed immunohistologically. All diagnoses were cytologically and histologically confirmed according to the WHO Nomenclature. The tumours were staged and graded as follows: patient 3- grade IV (poor) stage T3bN1bM0, patient 4- grade I (well) stage T2aNOM0, patient 5- grade I (well) stage T3bN1aM0, patient 6- grade I (well) stage T3bN1bM0, patient 7 - grade III (moderate) stage T3bN1bM0, patient 8- grade I (well) stage T1aN1bM0, patient 9- grade I (well) stage T2aNOM0. The non neoplastic control samples were collected from clinically unaltered tongue and palate tissues and the dogs were euthanized due to oral squamous cell carcinoma unrelated diseases. All samples were taken and provided by the Small Animal Clinic, University of Veterinary Medicine, Hannover, Germany according to the legislation of the state of Lower Saxony, Germany. 


\section{Generation of canine and human cell lines}

Due to the possibility to access fresh neoplastic material of both species we decided to aim at an establishment of OSCC cell lines as tools for further experimental approaches. The successful establishment of new cell lines allowed us to compare the gene expression patterns of the neoplastic primary tissues and the cell lines of both species. The respective human and canine tumour samples were verified to be squamous cell carcinomas by routine histopathologic characterisation. The samples were analysed by either a human or veterinary pathologist respectively. Two human cell lines were generated from freshly isolated squamous cell carcinoma biopsies derived from patient 4 and patient 12 (tumour staging see above). Single cell suspensions were prepared with a gentleMACS ${ }^{\text {тм }}$ tissue dissociator (Miltenyi Biotec GmbH, Bergisch Gladbach, Germany). Samples were cut into small pieces of approximately $5 \mathrm{~mm}$, transferred to a $\mathrm{C}$ Tube (Miltenyi Biotec GmbH, Bergisch Gladbach, Germany) containing $5 \mathrm{ml}$ Dulbecco's modified eagle medium (DMEM PAA, Pasching, Austria) and subjected to the first homogenisation step. After the addition of 1500 Units collagenase I (Cell Systems, St. Katharinen, Germany) and 0,5 mg neutral peptidase (Cell Systems, St. Katharinen, Germany), samples were incubated for $40 \mathrm{~min}$ at $37^{\circ} \mathrm{C}$. Digested samples were subjected to a second homogenisation step followed by removal of tissue debris using a $70 \mu \mathrm{M}$ cell strainer (BD Biosciences, Heidelberg, Germany). The cells were washed twice with culture medium (DMEM, 10\% fetal calf serum, $20 \mathrm{mM}$ Hepes, $1000 \mathrm{IU} / \mathrm{ml}$ penicillin and $0.1 \mathrm{mg} / \mathrm{ml}$ streptomycin; all PAA, Pasching, Austria) and plated on $100 \mathrm{~mm}$ cell culture dishes (Greiner, Frickenhausen, Germany) with DMEM and incubated at $37 \mathrm{C}$ and $5 \% \mathrm{CO}_{2}$ until confluent.

The canine cell line was generated from a freshly isolated oral squamous cell carcinoma biopsy. Due to the limited amount of bioptic material this sample was not used in the primary tissue screenings. The tumour tissue sample was cut into small pieces with a sterile scalpel and treated with collagenase $(0.26 \%)$ for 2 hours at $37^{\circ} \mathrm{C}$. The dissociated cells were transferred into a sterile $10 \mathrm{ml}$ tube and centrifuged for $10 \mathrm{~min}$ at $1000 * \mathrm{~g}$. After centrifugation the supernatant was discarded and the resuspended cell pellet transferred into a sterile flask and incubated in $5 \mathrm{ml}$ culture medium (Medium 199 (Invitrogen, Frankfurt, Germany), 10\% fetal calf serum (PAA, Pasching, Austria) $200 \mathrm{U} / \mathrm{ml}$ penicillin and $200 \mathrm{ng} / \mathrm{ml}$ streptomycin (Biochrom, Berlin, Germany) and incubated at $37^{\circ} \mathrm{C}$ and $5 \% \mathrm{CO}_{2}$ until confluent.

\section{Homogenisation of tissue samples and cell lysates of cultured cells}

Tissue samples were homogenised using the stainless steelbeads and Qiagen-TissueLyser II homogeniser method accordingly to the manufacturer's instructions (Qiagen,
Hilden, Germany). Lysates of cultured cells were homogenised with QIAshredder columns accordingly to the manufacturer's protocol (Qiagen, Hilden, Germany).

\section{RNA isolation and cDNA syntheses}

RNA from tissue samples and cultured cells was isolated using the RNeasy Mini Kit according to the manufacturer's instructions (Qiagen, Hilden, Germany). On-column DNase digestion was performed with the RNase-Free DNase set (Qiagen, Hilden, Germany). cDNA syntheses was performed using $250 \mathrm{ng}$ RNA and the QuantiTect Reverse Transcription Kit following the manufacturer's protocol (Qiagen, Hilden, Germany).

Furthermore, total RNA including small RNAs like miRNAs was isolated using the mirVana miRNA Isolation Kit according to the manufacturer's instructions (Ambion, Applied Biosystems, Darmstadt, Germany). The respective cDNA syntheses were performed using 100 ng total RNA of each sample and the TaqMan MicroRNA Reverse Transcription Kit following the manufacturer's protocol (Applied Biosystems, Darmstadt, Germany).

HMGA1, HMGA2, Lin28, GUSB and HPRT real time PCR

Relative quantification real time PCRs for both species were carried out using the Eppendorf Mastercycler ep realplex real-time PCR System (Eppendorf AG, Hamburg, Germany).

For analysis of the human target genes, $2 \mu \mathrm{l}$ of each cDNA was amplified in a total volume of $25 \mu \mathrm{l}$ using universal PCR Mastermix and commercially purchased TaqMan gene Expression Assays (HMGA1- Assay ID: Hs00600784_g1; HMGA2- Assay ID: Hs00971724_m1; Lin28A- Assay ID: Hs04189307_g1; HPRT- Assay ID: Hs02800695_m1; GUSB- Assay ID: Hs99999808_m1; (Applied Biosystems, Darmstadt, Germany)).

For analysis of canine target genes, $2 \mu \mathrm{l}$ of each cDNA was amplified using universal PCR Mastermix, self-designed TaqMan based Assays ([9,22] (canine HMGA1 (NM_001003387)- forward primer: 5' ACCC AGTGAAGTGCCAACACCTAA 3', reverse primer: 5' CCTCCTTCTCCAGTTTTTTGGGTCT 3', probe: 5' 6-FAM-AGGGTGCTGCCAAGACCCGGAAAACT ACCA-TAMRA 3'; canine HMGA2 (DQ316099)- forward primer: 5' AGTCCCTCCAAAGCAGCTCAAAAG 3', reverse primer: 5' GCCATTTCCTAGGTCTGCCTC 3', probe: 5' 6-FAM-CGCCCACTACTATGCCATCGT GTG-TAMRA 3'; canine HPRT (NM_001003357)- forward primer: 5'CCTTCTGCAGGAGAACCT 3', reverse primer: 5'TCATCACTAATCACGACGCT 3', probe: 5'6-FAMCCTCCTGTTCAGGCTGCCGTCA-TAMRA 3'; canine GUSB (NM_001003191)- forward primer: 5' TGGTGCT GAGGATTGGCA 3', reverse primer: 5' CTGCCACATG GACCCCATTC 3', probe: 5' 6-FAM-CGCCCACTA CTATGCCATCGTGTG-TAMRA 3') and commercially 
purchased TaqMan gene Expression Assays (Lin28- Assay ID: Cf02725509_g1 (Applied Biosystems, Darmstadt, Germany)).

The canine and human HMGA1 qPCR assays detected both splicing variants (HMGA1a and HMGA1b) simultaneously. PCR conditions were as follows: $10 \mathrm{~min}$ at $95^{\circ} \mathrm{C}$, followed by 45 cycles with $15 \mathrm{~s}$ at $95^{\circ} \mathrm{C}$ and $1 \mathrm{~min}$ at $60^{\circ} \mathrm{C}$. All human and canine samples were measured in triplicate and for each run non-template controls and non-reverse transcriptase control reactions were included.

\section{Let-7a, mir-98 and RNU6B real-time PCR}

Relative quantification of the human and canine let-7a, mir-98 and RNU6B micro RNA transcript levels were carried out using $1.33 \mu \mathrm{l}$ of each cDNA amplified in a total volume of $20 \mu \mathrm{l}$ using TaqMan Universal PCR Master Mix, No AmpErase UNG and TaqMan MicroRNA assays for each gene (Let-7a- Assay ID: 000377; mir-98- Assay ID: 000577; RNU6B- Assay ID: 001093 (Applied Biosystems, Darmstadt, Germany)).

PCR conditions were as follows: $10 \mathrm{~min}$ at $95^{\circ} \mathrm{C}$, followed by 45 cycles with $15 \mathrm{~s}$ at $95^{\circ} \mathrm{C}$ and $1 \mathrm{~min}$ at $60^{\circ} \mathrm{C}$. All samples were measured in triplicate and for each run non-template controls and non-reverse transcriptase control reactions were included.

A precedent efficiency analysis of the microRNA PCR assays which were used in this study was performed by applying the same template and dilution steps.

\section{Histological and immunohistochemical procedures}

The formalin-fixed specimens were embedded in paraffin, sectioned at $4 \mu \mathrm{m}$ and routinely stained with haematoxylin and eosin (H\&E). Thereafter, polyclonal goat-anti human HMGA2 (R\&D Systems, Minneapolis, MN, USA) (1:400) or mouse-anti human Ki-67 antibody (Dianova, Hamburg, Germany) (1:100) was applied and allowed to incubate for approximately $16-18 \mathrm{~h}$. Sections were incubated with biotin-conjugated horse antibody to goat IgG or goat anti-mouse IgG (both Vector Laboratories, Burlingame, CA, USA) (1:200) followed by ABC solution (Vectastain Elite $\mathrm{ABC}$ kit, Vector). Tyramine amplification reaction was performed according to the method of Adams (1992) [23] for 15 min (only HMGA2). The chromogen 3-amino-9-ethyl-carbazol (AEC) (Biologo, Kronshagen, Germany) was used for visualization followed by counterstaining with Mayer's haematoxylin. Negative control sections were prepared by substituting the primary antibody with PBS. For the scoring, the percentage of carcinoma cells with intense red positive nuclear labelling for HGMA2 was estimated by examining the centre of the tumour and the invasive front (0: no expression; $<25 \%$ weak expression; 25-50\% moderate expression; >50\%: strong expression).

\section{Statistical analysis}

Statistical analysis of the relative real time PCR results applying the Hypothesis Test was performed with the Relative expression software tool REST 2008, version 2.0.7 [24]. A p-value of $<0.05$ was considered statistically significant.

\section{Results}

Real time PCR expression analyses of HMGA1 and HMGA2 Human samples

HMGA1/GUSB expression levels varied from 0.225 to 1.47 within the control samples, and from 0.53 to 2.52 within the tumour samples. The HMGA1/HPRT expression levels ranged from 0.21 to 2.02 within the control samples and from 0.36 to 1.28 within the tumour samples (details Figure 1A, B and Additional file 1: Table S1).

HMGA2/GUSB values ranged between 1 and 26.8 in the control samples, 44.4 and 2330 in the tumour samples, and 319 and 1092 within the cell culture samples. HMGA2/ HPRT expression levels ranged between 1 and 31.1 within the control group, 24.1 and 3778 within the tumour group, and 226 to 561 within the cell culture samples (Figure 1C, D and Additional file 1: Table S1).

\section{Canine samples}

HMGA1/GUSB expression levels varied from 1 to 1.67 within the non-neoplastic samples and from 0.152 to 1.69 within the neoplastic samples. HMGA1/HPRT expression ranged from 1 to 1.31 in the non neoplastic and 0.269 to 0.811 within the tumour samples (details Figure 2A, B and Additional file 2: Table S2).

HMGA2/GUSB expression levels ranged from 0.383 to 1 within the non neoplastic samples and from 6.45 to 208 within the neoplastic samples. HMGA2/HPRT expression ranged from 0.31 to 1 within the control group, and from 3.69 to 80.7 within the tumours (Figure 2C, D and Additional file 2: Table S2).

\section{Statistical analyses of HMGA1 and HMGA2 expression Human samples}

HMGA1 was up-regulated when GUSB was used as endogenous control gene within the tumour samples $(\mathrm{p}=0.0011)$ when compared to the non neoplastic samples. HMGA2 was up-regulated when GUSB and $H P R T$ were used as endogenous control genes within the tumour and cell line samples when compared to the non neoplastic samples.

The respective $\mathrm{p}$-values of the tumour and cell line sample groups were $\mathrm{p}=0.000$ despite of the cell line sample group within the HMGA2/GUSB real time PCR showing a p-value of 0.001 (Figure 1).

\section{Canine samples}

HMGA1 was down-regulated when HPRT was used as endogenous control gene within the tumour samples 


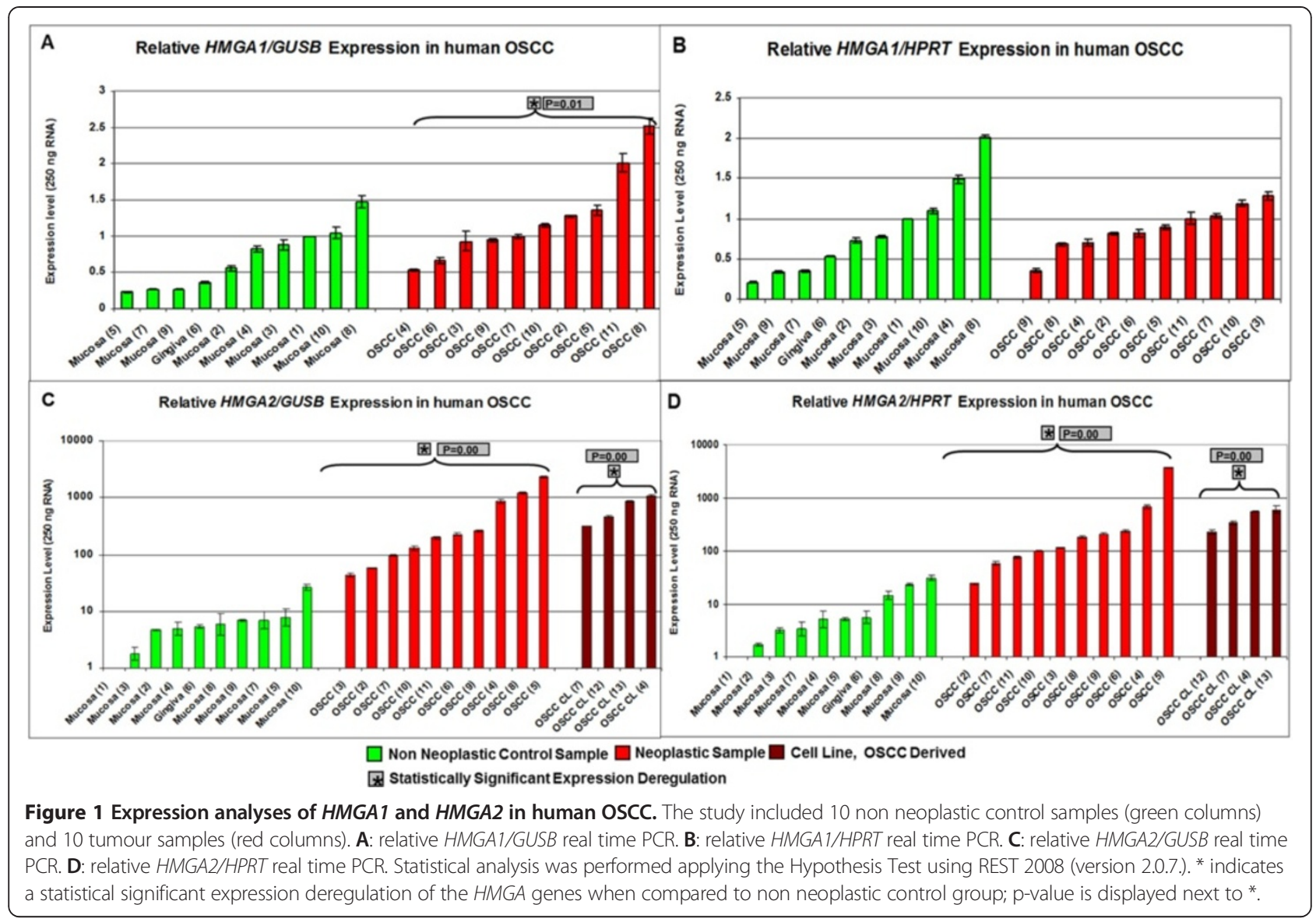

$(\mathrm{p}=0.0011)$ compared to the non neoplastic control samples. HMGA2 was up-regulated when GUSB and $H P R T$ were used as endogenous control genes within the tumour samples when compared to the non neoplastic controls. The analysed $\mathrm{p}$-values were $\mathrm{p}=0.002$ and $\mathrm{p}=0.003$ within the HMGA2/GUSB and HMGA2/HPRT real time PCRs respectively (Figure 2).

\section{Immunohistochemistry}

\section{Human tumour sections}

In the sections of patients 4 and 5 positive nuclear staining for HMGA2 was detected in the tumour cells that were located in the centre and/or the invasive front of the tumours (Figure 3A-C). The percentages of labelled tumour cells located in the centre were as follows: patient $4-30 \%$, patient $5-80 \%$. Staining intensities of HMGA2 of tumour cells located at the invasive front were as follows: patient $4-$ moderate $(2+)$, patient $5-$ strong $(3+)$. The remaining analysed sections of patients analysed did not show positive staining for HMGA2.

\section{Canine tumour sections}

In all sections positive nuclear staining for HMGA2 was detected in the tumour cells that were located in the centre and/or at the invasive front of the tumours as well (Figure 4). The percentages of labelled tumour cells located in the centre were as follows: patients 3, 4, 7, $8-25-50 \%$ respectively. Patients 5, 6 and $9-<25 \%$ respectively. The percentages of labelled tumour cells located at the invasive front were as follows: patient $3,5,6,8-25-50 \%$, patient 4, 7 and $9->50 \%$, respectively. Except the cases of patients 3 and 8, higher percentages of positive nuclei were found at the invasive front of the tumours. The percentages of Ki-67 labelled tumour cells at the invasive front varied between $36 \%$ and $68 \%$. In the decalcified sample from patient 6 , no Ki-67 labelling was seen.

\section{Comparative expression analyses of HMGA2, Lin28, let-7a} and mir-98

\section{Human samples}

Relative HMGA2/HPRT expression levels ranged from 1 to 4.61 within the non neoplastic samples, from 5.92 to 141 within the neoplastic samples and from 22.2 to 55.1 within the cell line samples (Figure 5A, Additional file 3: Table S3).

Lin 28/HPRT expression varied between 0 and 4.65 within the non neoplastic samples, from 0 to 27.5 within the neoplastic samples and from 1.03 to 4.87 


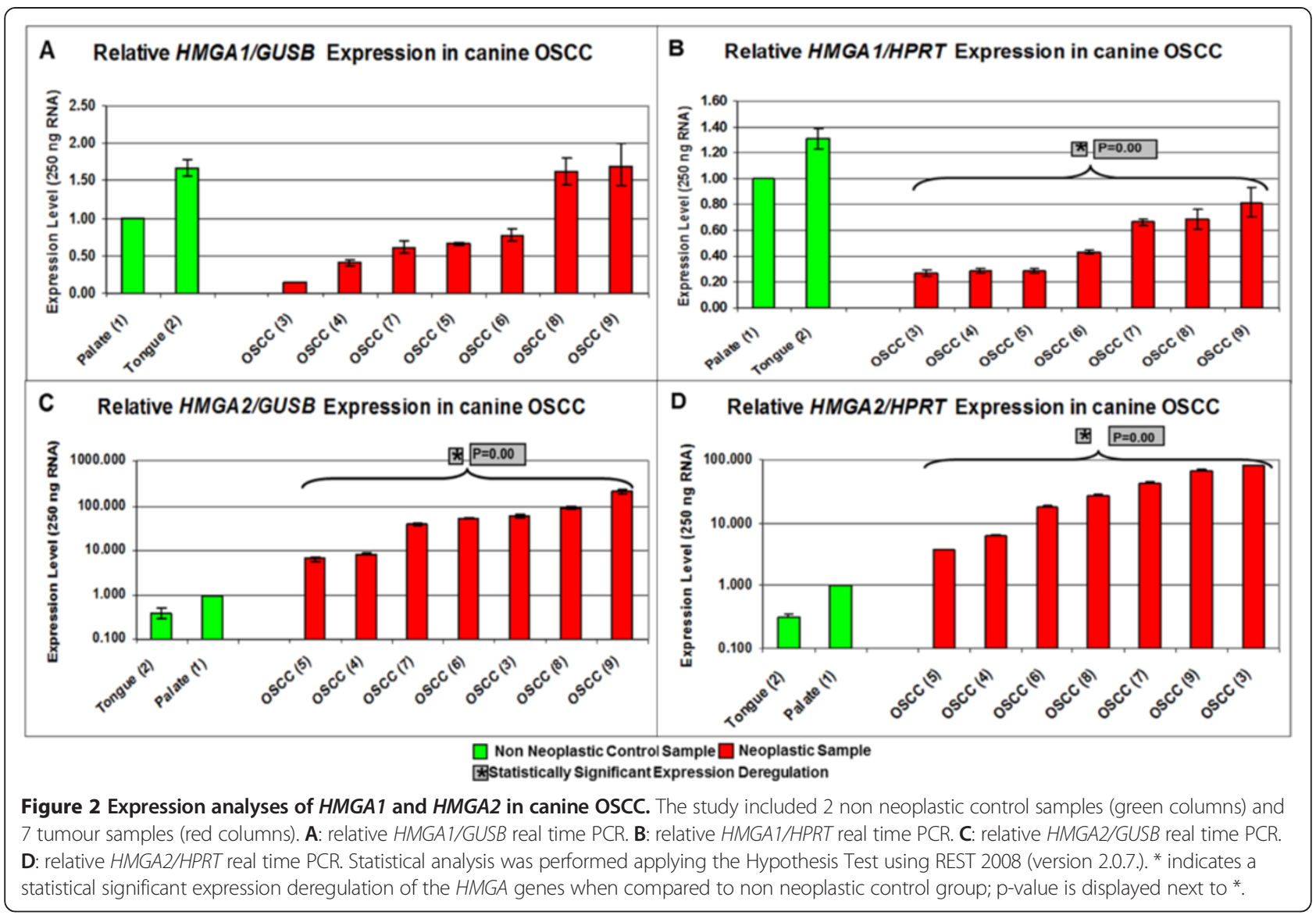

(patient 7) within the cell lines (Figure 5B, Additional file 3: Table S3).

let-7a/RNU6B expression ranged from 1 to 2.76 within the non neoplastic samples, from 1.14 to 3.42 within the neoplastic samples and from 1.11 to 2.33 within the cell lines (Figure 5C, Additional file 3: Table S3).

mir-98/RNU6B expression ranged from 1 to 2.28 within the non neoplastic samples, from 0.91 to 7.22 within the neoplastic and from 0.65 to 1.05 within the cell lines (Figure 5D, Additional file 3: Table S3).

\section{Canine samples}

HMGA2/HPRT expression ranged from 1 to 1.19 within the non neoplastic samples, from 3.09 to 178 within the neoplastic samples and from 242 to 270 within the cell line (Figure 6A, Additional file 4: Table S4).

Lin28/HPRT expression ranged between 0.02 and 1 within the non neoplastic samples, between 0 and 6.97 within the neoplastic samples, and between 0 and 0.03 within the cell line (Figure 6B, Additional file 4: Table S4).

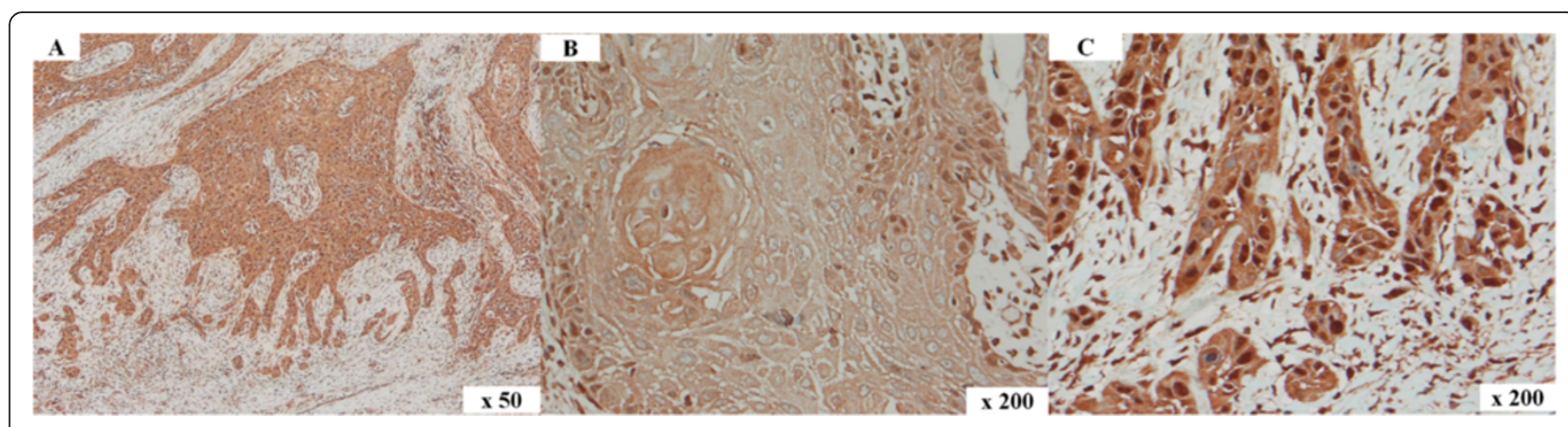

Figure 3 HMGA2 immunohistochemistry in human OSCC. Immunolabelling of a human tumour: overview (A), tumour centre (B) and invasive front (C). In the tumour centre (B) lower numbers of tumour cells with nuclear immunolabelling are present when compared to the respective invasive front (C). The invasive front shows numerous tumour cells exhibiting intense nuclear immunolabelling of HMGA2. Magnification: (A) 50x, (B) and (C) 200x. 


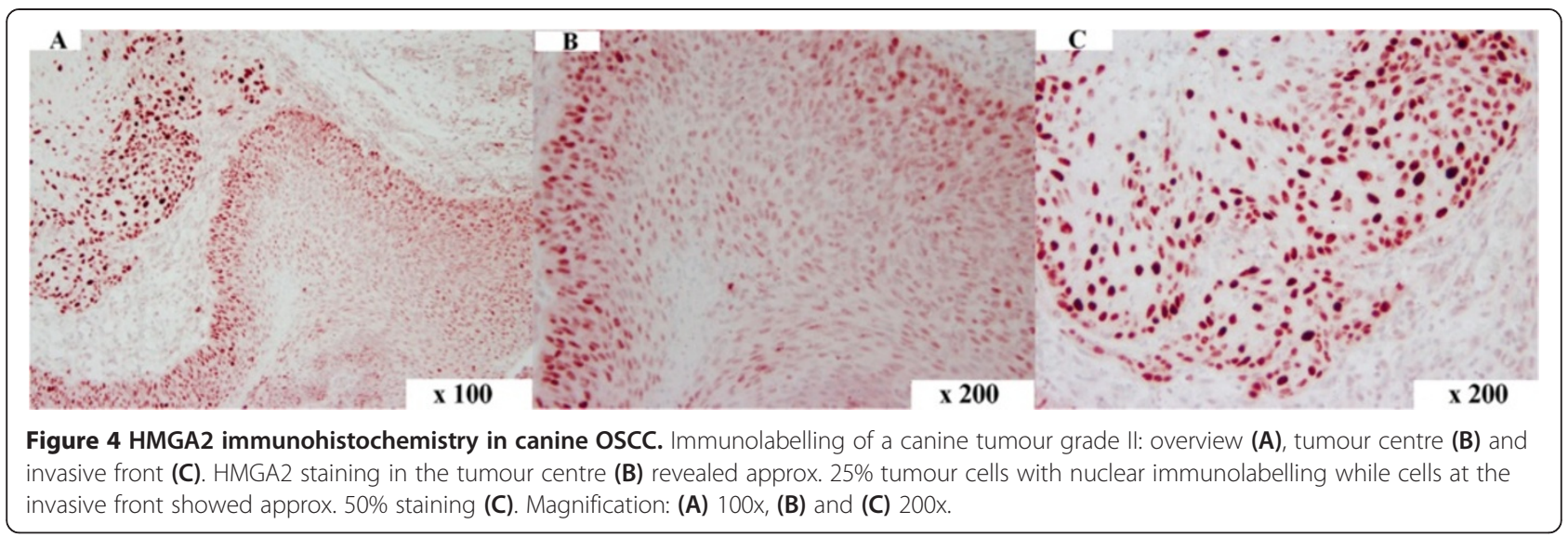

let7-a/RNU6B expression levels ranged from 1 to 2.32 within the non neoplastic samples, from 0.84 to 7.36 within the neoplastic samples and from 5.6 to 11.7 within the cell line samples (Figure 6C, Additional file 4: Table S4).

Mir-98/RNU6B expression ranged from 1 to 3.8 within the non neoplastic samples, from 3.93 to 6.97 within the neoplastic samples and from 4.06 to 5.18 within the cell line (Figure 6D, Additional file 4: Table S4).
Statistical analyses of comparative HMGA2, Lin28, let-7a and mir-98 expression

Human samples

HMGA2 was up-regulated when HPRT was used as endogenous control gene within the tumour $(\mathrm{p}=0.002)$ and cell line samples $(\mathrm{p}=0.024)$ when compared to the non neoplastic samples (Figure 5A). mir-98 was down-regulated when RNU6B was used as endogenous

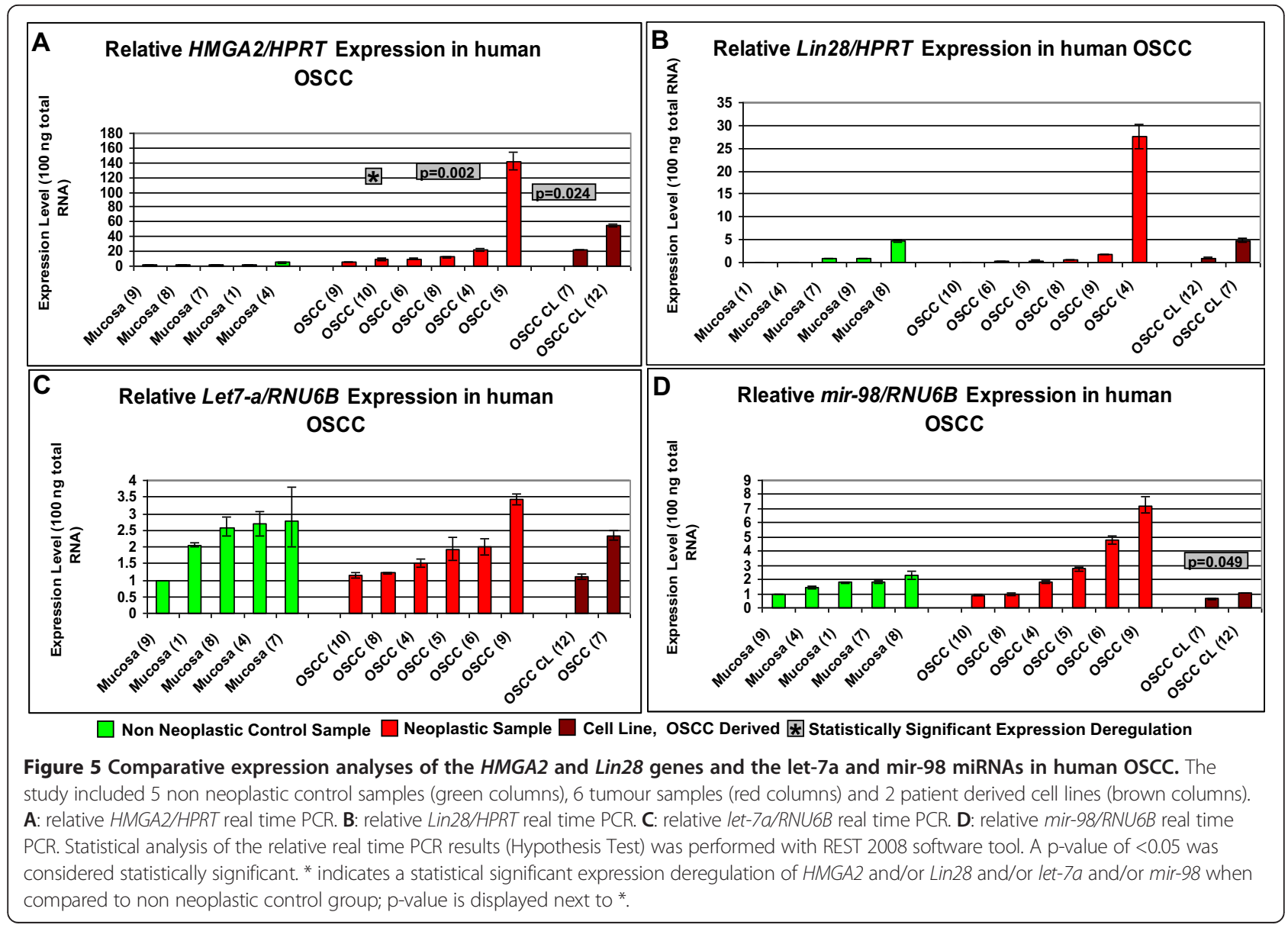



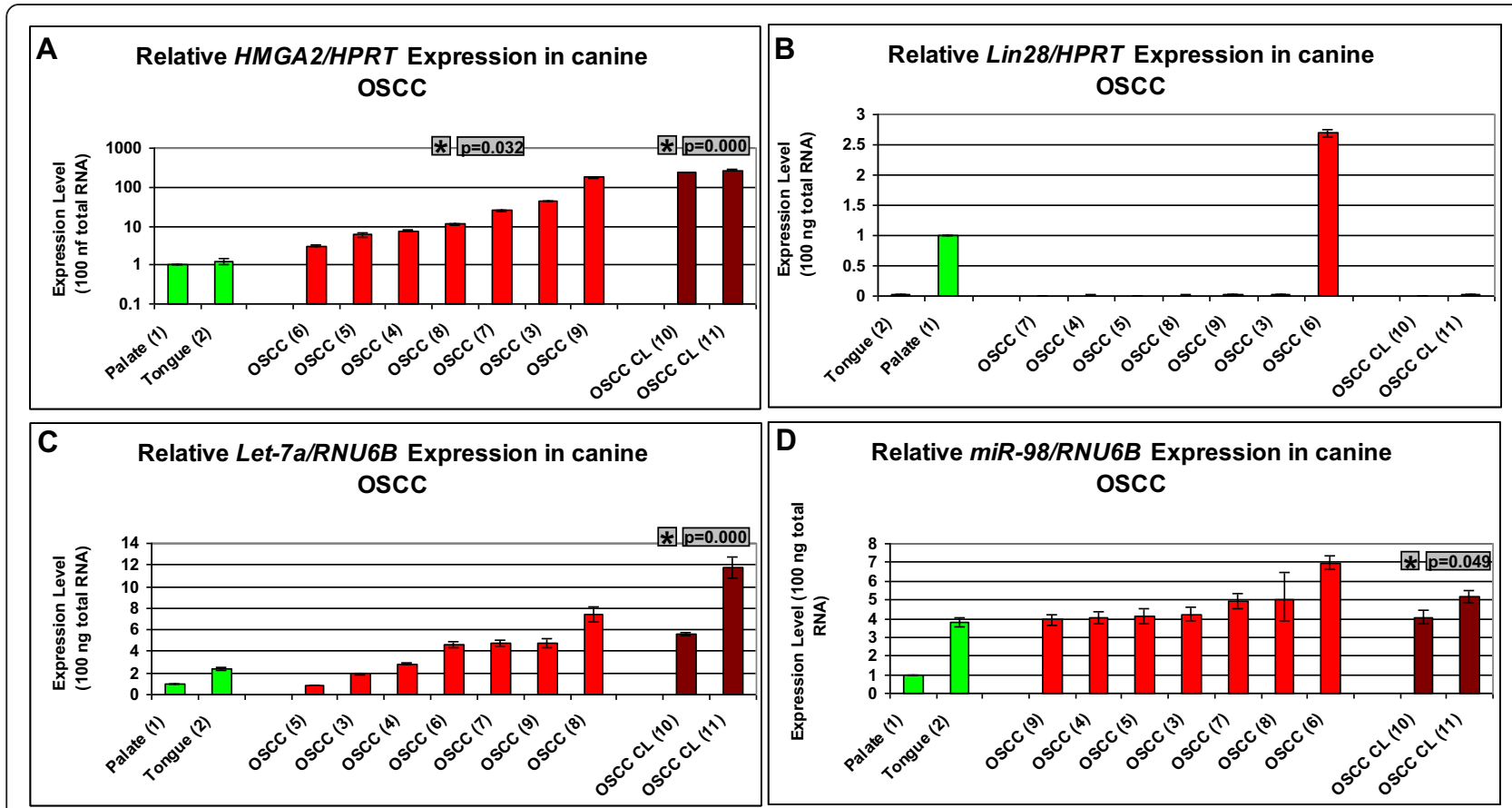

$\square$ Non Neoplastic Control Sample $\square$ Neoplastic Sample $\square$ Cell Line, OSCC Derived 因 Statistically Significant Expression Deregulation

Figure 6 Comparative expression analyses of the HMGA2 and Lin28 genes and the let-7a and mir-98 miRNAs in canine OSCC. The study included 2 non neoplastic control samples (green columns), 7 tumour samples (red columns) and 2 cell line derived samples (brown columns) which derived from patients 1-10. A: relative HMGA2/HPRT real time PCR. B: relative Lin28/HPRT real time PCR. C: relative let-7a/RNU6B real time PCR. D: relative mir-98/RNU6B real time PCR. Statistical analysis of the relative real time PCR results (Hypothesis Test) was performed with REST 2008 software tool. A p-value of $<0.05$ was considered statistically significant. * indicates a statistical significant expression deregulation of HMGA2 and/or Lin28 and/or let-7a and/or mir-98 when compared to non neoplastic control group; $\mathrm{p}$-value is displayed next to *

control within the cell line samples $(\mathrm{p}=0.049)$ when compared to the non neoplastic control samples (Figure 5D).

\section{Canine samples}

HMGA2 was up-regulated when HPRT was used as endogenous control gene within the tumour $(\mathrm{p}=0.032)$ and cell line samples $(\mathrm{p}=0.000)$ when compared to the non neoplastic samples (Figure 6A). let7-a was up-regulated when RNU6B was used as endogenous control within the cell lines samples $(p=0.000)$ when compared to the non neoplastic control samples (Figure 6C). mir-98 was up-regulated when RNU6B was used as endogenous control within the tumour samples $(\mathrm{p}=0.000)$ when compared to the non neoplastic control samples (Figure 6D).

\section{Discussion}

The evaluation of the HMGA gene expression in the analysed human and canine neoplasias showed consistent expression in both species. Whereas HMGA2 was found to be strongly upregulated in the primary samples as well as in the analysed cell lines the sister gene HMGA1 showed expression variation ranging from two to three fold. Thus, herein HMGA2 expression shows potential marker characteristics in both species whereas HMGA1 misses the required characteristics.

Previous studies analysing the HMGA1 expression in human head and neck cancers reported contradictory results, showing no statistical significant deregulations as well as increased expression of HMGA1 in OSCC [11,12], matching our results in both species. The herein characterised high HMGA2 expression in the neoplastic samples of both species, affirms the findings by Miyazawa et al. [11] reporting HMGA2 over-expression of 84-315-fold in analysed carcinoma tissues when compared to non neoplastic tissues [11].

HMGA2 was found to be expressed at the invasive front of oral carcinomas leading to the conclusion that - in contrast to HMGA1- HMGA2 immunostaining could be a potential prognostic determinant in stratifying patients into risk groups [11]. Further, multivariate risk factor analysis demonstrated that HMGA2 expression was found to be a significant independent predictor of death of carcinoma and as an independent prognostic marker for disease-specific overall survival [11]. Contrary to this, HMGA1 expression was also reported to be increased in head and neck carcinomas analysed via 
semi-quantitative RT-PCR and immunohistochemistry when compared to healthy mucosa samples [12].

The herein reported immunohistochemical staining in human sections showed positive HMGA2 staining within the centre and the invasive front of the tumour sections in only two patients. In contrast, in all canine samples the immunohistochemichal staining showed positive signals for HMGA2. Thereby, with exception of two cases, the percentages of positive nuclei for HMGA2 were higher at the invasive front of the tumour sections than in the centre of the tumours. Similarly, Miyazawa et al. reported a negligible HMGA2 staining in the central area of the human carcinoma tissues whereas it was ectopically expressed at the invasive front [11]. In general, most of the oral cancer deaths result from local invasion and distant metastasis. At the invasive front, the carcinoma cells gain the characteristics similar to mesenchymal cells due to epithelial-mesenchymal transition (EMT) where cells switch from a polarised epithelial phenotype to a motile mesenchymal phenotype and facilitate tumour invasion $[25,26]$. The role of HMGA2, which is a mesenchymspecific factor was thus strongly correlated with poor differentiation, invasion and metastasis of oral cancer $[11,25,26]$. Our immunohistochemical findings in canine OSCC are comparable with the findings seen in human OSCC supporting the data discussing a possible role of HMGA2 as a factor promoting cell proliferation and motility at the invasive front in canine oral cancer.

In our study, mir-98 was significantly down-regulated in the analysed OSCC cell lines describing an inverse expression pattern of HMGA2 and mir-98. As this observation is limited to $\mathrm{qPCR}$ assays, a definitive statement can hardly be drawn at this point without further transcriptomic analyses. However, interestingly Hammond et al. postulated a linear pathway from Lin28 to let-7 to HMGA2 [27]. In embryonic stem cells Lin28 expression is highly leading to the inhibition of let-7 microRNAs processing steps. These low let-7 microRNAs levels allow a high expression of their usually repressed target genes as HMGA2. Accordingly, HMGA2 expression increases as observed in embryonic stem cells and in several cancers [27]. Our results are in agreement with a study by Hebert et al. reporting that HMGA2 expression is partly regulated by mir- 98 in head and neck squamous cell carcinoma cell lines [13]. The let-7a miRNA was reported to be down-regulated in head and neck cancer tissues and the expression levels were significantly reduced in metastatic tissues when compared to primary tumours. Thereby, a high let-7a score was found to be associated with early $\mathrm{T}$-stage and low lymph node metastasis and early pathological stage [28]. Additionally, in human oesophageal squamous cell carcinoma, an inverse transcription of let-7 and HMGA2 was reported [29]. However, the authors did not mention which members of the let-7 family were analysed and thus it cannot be excluded that let-7a and mir-98 were not analysed within the study.

We performed the statistical analysis of the different real time PCRs for all samples as groups with no regard to the single patients. The human patients 4 and 7 could provide real time data for both sample types as non-neoplastic and neoplastic material was existent. Expression of $H M G A 2$ and $\operatorname{Lin} 28$ were higher in the neoplastic samples while let-7a and mir-98 expression were higher in the non-neoplastic samples. Here, an inverse correlation of the expression of HMGA2 and Lin 28 and let-7a and mir-98 could be detected in the tumour samples when compared to healthy tissue. These results strongly fortify the described $\operatorname{Lin} 28$ to let-7 to HMGA2 axis hypothesis drawn by Hammond et al. [27]. Further, increased levels of Lin $28 b$ could be associated with poor prognosis OSCCs [21]. Nevertheless, it must be considered that the statistical analyses of our real time PCRs did not confirm a significant down-regulation of let-7a and a simultaneous over-expression of $\operatorname{Lin} 28$ when all samples were analysed as groups. In general, an inverse correlation of let-7 and HMGA2 is a frequent finding in many types of human neoplasias. Nevertheless, in adipocytic tumours, despite of some individual let-7 miRNAs, no global correlation between expression of let-7 members and HMGA2 were detected [30]. Similarly, our results regarding let-7a expression suggest, that a decrease in let-7a expression does not appear to be main mechanism accounting for HMGA2 deregulation. Hereby, let-7a is likely to be involved in the pathogenesis of OSCC probably through mechanisms other than those expected.

Nevertheless, our results provide a first trend of comparative expression of HMGA2, Lin28, let-7a and mir-98 in OSCC and must be verified in a larger set of tissue control and tumour samples to determine if there is an inverse correlation of expression between these genes and if there is a potential link to disease progression.

Comparative expression analyses of $H M G A 2, \operatorname{Lin} 28$, let-7a and mir-98 in canine samples showed a statistical significant over expression of HMGA2 in neoplastic samples confirming our precedent results. Besides this, the study revealed different results when compared to the results made in the comparative expression study in human samples. Let-7a was over expressed within the cell line samples while mir-98 was over expressed in the tumour samples. These findings do not indicate a negative correlation of expression of HMGA2 and Lin28 and the let-7a and mir-98. Thus, analysis of more control and tumour samples would be required to improve the power of the study and additional microRNAs should be taken into account, which might be involved in the progression of canine OSCC. 
A correlation between the analysed targets and the survival time was not focussed in this study due to the limited number of analysed cases. Additionally, regarding canine patients, an accurate follow up is often hindered by the fact that veterinary patients are frequently not represented at the academic institution as owners follow treatment at local veterinarians. Furthermore, central cancer register for canine patients, as present for human cancer patients, does not exist. However, due to the similarities in canine and human cancer presentation, as reported herein, basic research and the development of clinical regimens in either of the species provide valuable solid data for the respective counterpart.

\section{Conclusion}

In conclusion, the comparative expression study analysing a possible inverse correlation between HMGA2 and $\operatorname{Lin} 28$ and let-7 members in OSCC revealed a down-regulation of mir-98 with a simultaneous HMGA2 over expression in human OSCC cell line samples. In contrast to the findings made in human samples, comparative analyses in canine showed different expression patterns. Despite the HMGA2 over-expression in neoplastic samples, the miRNAs let-7a and mir-98 were found to be up-regulated. Consequently, in canine OSCC further factors might be involved in the progression neoplasia when compared to the analysed human OSCCs. Furthermore the miRNAs analysed herein might not reflect the main factors involved in deregulation of HMGA2 in canine OSCC.

\section{Additional files}

Additional file 1: Table S1. Expression analyses of HMGA1 and HMGA in human OSCC. Relative real-time PCR reactions were performed with human GUSB and HPRT as endogenous control genes. The non neoplastic mucosa sample obtained from patient 1 was used for calibration during data analyses.

Additional file 2: Table S2. Expression analyses of HMGA1 and HMGA2 in canine OSCC. Relative real-time PCR reactions were performed with canine GUSB and HPRT as endogenous control genes. The non neoplastic palate sample obtained from patient 1 was used for calibration during data analyses.

Additional file 3: Table S3. Comparative expression analyses of the HMGA2 and Lin28 genes and the let-7a and mir-98 miRNAs in human OSCC Relative real-time PCR reactions were performed with human HPRT and RNU6B as endogenous control genes. The non neoplastic mucosa sample obtained from patient 9 was used for calibration during data analyses.

Additional file 4: Table S4. Comparative expression analyses of the HMGA2 and Lin28 genes and the let-7a and mir-98 miRNAs in canine OSCC Relative real-time PCR reactions were performed with canine HPRT and RNU6B as endogenous control genes. The non neoplastic palate sample obtained from patient 1 was used for calibration during data analyses.

\section{Competing interests}

The authors declare that they have no competing interests.

\section{Authors' contributions}

KAS: All nucleic acid work, QPCR, data analyses, partial manuscript drafting AE: coordination and supervision of human cell culture, partial study design, human surgery and sample collection, partial manuscript drafting. AK: establishment of human cell lines, partial manuscript drafting. SW: establishment of canine cell line, manuscript editing. NE: sampling of canine tissues. FL: IHC human samples, data analyses, partial manuscript drafting. SK: IHC canine samples, data analyses, partial manuscript drafting. MHT: IHC canine samples, data analyses, manuscript editing. HK: head of the Institute for Pathology, partial study design, IN: initiated the study, partial study design, approved the final manuscript. HME: principal study design, coordination and supervision of canine cell culture and complete nucleic acid work, partial manuscript drafting and finalization, NCG: head of the Department of Oral and Maxillofacial Surgery, performed partial study design, approved the final manuscript. All authors read and approved the final manuscript.

\section{Acknowledgements}

H. Murua Escobar thanks Dr. Frank Conradi (Bremen) for fruitful discussion.

\section{Author details}

'Small Animal Clinic, University of Veterinary Medicine Hannover, Bünteweg 9, 30559 Hannover, Germany. ${ }^{2}$ Department of Oral and Maxillofacial Surgery, Hannover Medical School, Carl-Neuberg-Strasse 1, 30625 Hannover, Germany. ${ }^{3}$ Institute for Pathology, Hannover Medical School, Carl-Neuberg-Strasse 1, 30625 Hannover, Germany. ${ }^{4}$ Department of Pathology, University of Veterinary Medicine Hannover, Bünteweg 17, 30559 Hannover, Germany. ${ }^{5}$ Division of Medicine, Department of Haematology/Oncology, University of Rostock, Ernst-Heydemann-Strasse 6, 18057 Rostock, Germany.

Received: 27 March 2013 Accepted: 17 September 2014

Published: 23 September 2014

\section{References}

1. Petersen PE: Oral cancer prevention and control-the approach of the World Health Organization. Oral Oncol 2009, 45(4-5):454-460.

2. Mannarini L, Kratochvil V, Calabrese L, Gomes Silva L, Morbini P, Betka J, Benazzo M: Human Papilloma Virus (HPV) in head and neck region: review of literature. Acta Otorhinolaryngol Ital 2009, 29(3):119-126.

3. Withrow S, Vail D: Withrow and MacEwen's Small Animal Clinical Oncology. 4th edition. St. Louis: Saunders Elsevier; 2007.

4. Mallet $Y$, Avalos N, Le Ridant AM, Gangloff P, Moriniere S, Rame JP, Poissonnet G, Makeieff M, Cosmidis A, Babin E, Barry B, Fournier C: Head and neck cancer in young people: a series of 52 SCCs of the oral tongue in patients aged 35 years or less. Acta Otolaryngol 2009, 129(12):1503-1508.

5. Kokemueller H, Rana M, Rublack J, Eckardt A, Tavassol F, Schumann P, Lindhorst D, Ruecker M, Gellrich NC: The Hannover experience: surgical treatment of tongue cancer-a clinical retrospective evaluation over a 30 years period. Head Neck Oncol 2011, 3:27.

6. Nao EE, Dassonville O, Poissonnet G, Chamorey E, Pierre CS, Riss JC, Vincent N, Peyrade F, Benezery K, Chemaly L, Sudaka A, Marcy PY, Vallicioni J, Demard F, Santini J, Bozec A: Ablative surgery and free flap reconstruction for elderly patients with oral or oropharyngeal cancer: oncologic and functional outcomes. Acta Otolaryngol 2011, 131(10):1104-1109.

7. Williams MD: Integration of biomarkers including molecular targeted therapies in head and neck cancer. Head Neck Pathol 2010, 4(1):62-69.

8. Cleynen I, Van de Ven WJ: The HMGA proteins: a myriad of functions (Review). Int J Oncol 2008, 32(2):289-305.

9. Joetzke AE, Sterenczak KA, Eberle N, Wagner S, Soller JT, Nolte I, Bullerdiek J, Murua Escobar H, Simon D: Expression of the high mobility group A1 (HMGA1) and A2 (HMGA2) genes in canine lymphoma: analysis of 23 cases and comparison to control cases. Vet Comp Oncol 2010, 8(2):87-95.

10. Winkler S, Murua Escobar H, Meyer B, Simon D, Eberle N, Baumgartner W, Loeschke S, Nolte I, Bullerdiek J: HMGA2 expression in a canine model of prostate cancer. Cancer Genet Cytogenet 2007, 177(2):98-102.

11. Miyazawa J, Mitoro A, Kawashiri S, Chada KK, Imai K: Expression of mesenchyme-specific gene HMGA2 in squamous cell carcinomas of the oral cavity. Cancer Res 2004, 64(6):2024-2029. 
12. Rho YS, Lim YC, Park IS, Kim JH, Ahn HY, Cho SJ, Shin HS: High mobility group HMGI(Y) protein expression in head and neck squamous cell carcinoma. Acta Otolaryngol 2007, 127(1):76-81.

13. Hebert C, Norris K, Scheper MA, Nikitakis N, Sauk JJ: High mobility group A2 is a target for miRNA-98 in head and neck squamous cell carcinoma. Mol Cancer 2007, 6:5.

14. Mu G, Liu H, Zhou F, Xu X, Jiang H, Wang Y, Qu Y: Correlation of overexpression of HMGA1 and HMGA2 with poor tumor differentiation, invasion, and proliferation associated with let-7 down-regulation in retinoblastomas. Hum Pathol 2010, 41(4):493-502.

15. Rahman MM, Qian ZR, Wang EL, Sultana R, Kudo E, Nakasono M, Hayashi T, Kakiuchi S, Sano T: Frequent overexpression of HMGA1 and 2 in gastroenteropancreatic neuroendocrine tumours and its relationship to let-7 downregulation. Br J Cancer 2009, 100(3):501-510.

16. Heo I, Joo C, Cho J, Ha M, Han J, Kim VN: Lin28 mediates the terminal uridylation of let-7 precursor MicroRNA. Mol Cell 2008, 32(2):276-284.

17. Newman MA, Thomson JM, Hammond SM: Lin-28 interaction with the Let-7 precursor loop mediates regulated microRNA processing. RNA 2008, 14(8):1539-1549.

18. Viswanathan SR, Daley GQ, Gregory Rl: Selective blockade of microRNA processing by Lin28. Science 2008, 320(5872):97-100.

19. Bussing I, Slack FJ, Grosshans H: let-7 microRNAs in development, stem cells and cancer. Trends Mol Med 2008, 14(9):400-409.

20. Ji J, Wang XW: A Yin-Yang balancing act of the lin28/let-7 link in tumorigenesis. J Hepatol 2010, 53(5):974-975.

21. Wu T, Jia J, Xiong X, He H, Bu L, Zhao Z, Huang C, Zhang W: Increased expression of Lin28B associates with poor prognosis in patients with oral squamous cell carcinoma. PLoS One 2013, 8(12):e83869.

22. Sterenczak KA, Joetzke AE, Willenbrock $S$, Eberle $N$, Lange $S$, Junghanss $C$, Nolte I, Bullerdiek J, Simon D, Murua Escobar H: High-mobility group B1 (HMGB1) and receptor for advanced glycation end-products (RAGE) expression in canine lymphoma. Anticancer Res 2010, 30(12):5043-5048.

23. Adams JC: Biotin amplification of biotin and horseradish peroxidase signals in histochemical stains. J Histochem Cytochem 1992, 40(10):1457-1463.

24. Pfaffl MW, Horgan GW, Dempfle L: Relative expression software tool (REST) for group-wise comparison and statistical analysis of relative expression results in real-time PCR. Nucleic Acids Res 2002, 30(9):e36.

25. Chang JY, Wright JM, Svoboda KK: Signal transduction pathways involved in epithelial-mesenchymal transition in oral cancer compared with other cancers. Cells Tissues Organs 2007, 185(1-3):40-47.

26. Imai K: Epitehlial-mesenchymal transition and progression of oral carcinomas. Cancer Therapy 2004, 2:6.

27. Hammond SM, Sharpless NE: HMGA2, microRNAs, and stem cell aging. Cell 2008, 135(6):1013-1016.

28. Yu CC, Chen YW, Chiou GY, Tsai LL, Huang PI, Chang CY, Tseng LM, Chiou SH, Yen SH, Chou MY, Chu PY, Lo WL: MicroRNA let-7a represses chemoresistance and tumourigenicity in head and neck cancer via stem-like properties ablation. Oral Oncol 2011, 47(3):202-210.

29. Liu Q, Lv GD, Qin X, Gen YH, Zheng ST, Liu T, Lu XM: Role of microRNA let-7 and effect to HMGA2 in esophageal squamous cell carcinoma. Mol Biol Rep 2012, 39(2):1239-1246.

30. Bianchini L, Saada E, Gjernes E, Marty M, Haudebourg J, Birtwisle-Peyrottes I, Keslair F, Chignon-Sicard B, Chamorey E, Pedeutour F: Let-7 microRNA and HMGA2 levels of expression are not inversely linked in adipocytic tumors: analysis of 56 lipomas and liposarcomas with molecular cytogenetic data. Genes Chromosomes Cancer 2011, 50(6):442-455.

\section{Submit your next manuscript to BioMed Central and take full advantage of:}

- Convenient online submission

- Thorough peer review

- No space constraints or color figure charges

- Immediate publication on acceptance

- Inclusion in PubMed, CAS, Scopus and Google Scholar

- Research which is freely available for redistribution

Submit your manuscript at www.biomedcentral.com/submit 